\title{
Knockdown of mutated H-Ras V12 expression induces chemosensitivity of hepatocellular carcinoma cells to cisplatin treatment in vitro and in nude mouse xenografts
}

\author{
FANJIE MENG, BIN CAO, ZENGLI FENG, SHUNMAO MA, HAIGANG WANG, YANSHU LI and HUI LI
}

Department of General Surgery, North-China Oil Field General Hospital, Hebei Medical University, Renqiu 062552, P.R. China

Received May 14, 2014; Accepted August 26, 2014

DOI: $10.3892 /$ or.2014.3466

\begin{abstract}
Ras mutations contribute to human cancer development. The present study assessed the Ras V12 mutation in hepatocellular carcinoma (HCC) cells and the role of its silencing in vitro and in nude mouse xenografts. HCC BEL7402 cells expressed mutations of V12 (Val/Gly) and wild-type H-Ras, whereas SMMC7721 cells only expressed wild-type $H$-Ras. The shRNA constructs specifically silenced mutated H-Ras expression in BEL7402 cells, but did not affect wild-type Ras expression in SMMC7721 cells. Silencing of mutated H-Ras expression reduced BEL7402 cell viability, induced apoptosis and arrested cells at the G0/G1 phase of the cell cycle. Expression of phospho-Akt was also significantly decreased, while several apoptotic proteins were also activated. Furthermore, sensitivity of stably H1-shRNA and H2-shRNA transfected BEL7402 cells to cisplatin treatment was increased by 7.19- and 5.39-fold, respectively, compared to that in the negative control cells, and apoptosis-related gene expression was also altered. Intraperitoneal administration of cisplatin led to a substantial reduction in HCC xenograft growth by 81 and $77 \%$ in the H1-shRNA and H2-shRNA transfected tumor xenografts, respectively, compared to a $37 \%$ reduction in mice bearing negative control tumor cells. These data demonstrate that knockdown of mutated $H$-Ras V12 expression induced chemosensitivity of HCC cells to cisplatin treatment in vitro and in vivo.
\end{abstract}

\section{Introduction}

Hepatocellular carcinoma (HCC) is a significant worldwide health issue, accounting for more than 740,000 new cases and

Correspondence to: Dr Bin Cao, Department of General Surgery, North-China Oil Field General Hospital, Hebei Medical University, Renqiu 062552, P.R. China

E-mail: huayouwaike@163.com

Key words: H-Ras V12, hepatocellular carcinoma, chemosensitivity, cisplatin
695,500 cancer-related deaths worldwide according to 2008 statistical data. Approximately $85 \%$ of HCC cases occur in developing countries, such as China, which alone accounts for $50 \%$ of the total cases. The reason is thought to be due to epidemic infection of hepatitis $\mathrm{B}$ or $\mathrm{C}$ virus (HBV or $\mathrm{HCV}$ ). Prevention of HBV and HCV infection, reduced alcohol consumption, and implementation of policies to reduce aflatoxin contamination in the food supply could effectively reduce $\mathrm{HCC}$ incidence (1). Clinically, $\mathrm{HCC}$ is a very aggressive disease and the survival rate of HCC patients is still low, even with advancements in early detection, treatment options and liver transplantation. Thus, the development and identification of novel strategies are urgently needed to effectively control HCC mobility and mortality.

Towards this end, our research objective was to target Ras oncoprotein. The Ras family of proteins belongs to a class of small GTPases and is involved in cellular signal transduction by switching on and off to regulate a wide variety of cellular processes, including growth, differentiation, apoptosis, cytoskeletal organization and membrane trafficking (2-4). As a result, Ras mutations can lead to the production of permanently activated Ras oncoprotein, which occurs in $~ 30 \%$ of all human cancers $(4,5)$. In HCC, a recent study showed that H-Ras mutation was present in 49/69 (71.01\%) of HCC patient samples (6). Overall, the mutated Ras protein leads to uncontrolled cell proliferation and resistance to apoptosis signals (4-6). Thus far, Ras family members that have been reported to be mutated in different types of cancer include K-Ras in pancreatic, nonsmall cell lung and colorectal cancer and seminoma; N-Ras in melanoma, HCC, myelodysplastic syndrome and acute myelogenous leukemia; and H-Ras in follicular and undifferentiated papillary thyroid, bladder and renal cell cancer (7). Recently, targeting of Ras oncoprotein or signaling using Ras siRNA or small molecules has been researched in both in vitro studies and in clinical trials $(8,9)$. Published studies have shown some promising data (10) and other studies are still ongoing. For example, a current clinical trial is using refametinib to treat patients with unresectable or metastatic HCC carrying a $R A S$ mutation (NCI protocol no. NCT01915589). To date, pharmacological strategies are mainly focused on post-translational modification of Ras oncoprotein, such as farnesyltransferase and geranylgeranyltransferase inhibitors, 
and antisense RNA (11). As the Ras signaling pathway can be found in all cells, Ras inhibitors inevitably affect normal cells as well as tumor cells, and these non-specific approaches are therefore associated with more side-effects that cannot be ignored $(1,12)$. Thus, in the present study, we used vectormediated expression of short hairpin RNAs (shRNAs) against a specific H-Ras mutation in a HCC cell line and then assessed the modulation of $\mathrm{HCC}$ cell chemosensitivity in vitro and in vivo using nude mouse xenografts.

\section{Materials and methods}

Cell lines, culture and reagents. Human liver cancer cell lines, BEL7402 and SMMC7721, were obtained from the American Type Culture Collection (Manassas, VA, USA) and cultured in RPMI-1640 medium supplemented with $10 \%$ fetal calf serum (FCS). Cisplatin (DDP) was purchased from Sigma Chemical Co. (St. Louis, MO, USA), and antibodies against Akt, p-Akt (Ser473), Erk1/2, p-Erk1/2 (Thr202/ Tyr204), Bcl-2, p53 upregulated modulator of apoptosis (Puma), polyADP-ribose polymerase (PARP) and caspase-3 were obtained from Cell Signaling Technology, Inc. (Beverly, MA, USA).

RT-PCR and DNA sequencing for detection of the H-Ras mutation in HCC cell lines. Total cellular RNA from cells was isolated using the TRIzol reagent (Invitrogen, Carlsbad, CA, USA) and reversely transcribed into cDNA using a kit from Invitrogen, according to the manufacturer's instructions. The primers used for amplification of $\mathrm{H}$-Ras mRNA (NM_001130442.1) were: 5'-GCGCCTGTGAACGGTGG-3' and 5'-TGGGCACGTCAT CCGAGTCC-3' to amplify a 397-bp PCR product. The PCR conditions were as following: an initial hot denaturation at $94^{\circ} \mathrm{C}$ for $15 \mathrm{~min}$ and then 35 cycles of denaturing at $94^{\circ} \mathrm{C}$ for $20 \mathrm{sec}$, annealing at $62^{\circ} \mathrm{C}$ for $20 \mathrm{sec}$, extension at $72^{\circ} \mathrm{C}$ for $30 \mathrm{sec}$ and a final step at $72^{\circ} \mathrm{C}$ for $10 \mathrm{~min}$. To verify the amplification of the mutant sequences, PCR products were purified and subjected to direct sequencing.

Construction of the H-Ras shRNA vector and gene transfection. To knock down H-Ras V12 expression in HCC cell lines, we first designed two siRNA constructs using the 'siRNA Target Finder' on the Ambion (Austin, TX, USA) company website (schematically illustrated in Fig. 1C). The oligonucleotides containing sense and antisense sequences were synthesized by Takara Biotechnology Co., Ltd. (Dalian, China). To construct the vector, $20 \mu \mathrm{M}$ of each sense and antisense oligonucleotides were annealed in 10X buffer $(100 \mathrm{mM}$ Tris-HCl, pH 7.5, $1 \mathrm{M} \mathrm{NaCl}, 10 \mathrm{mM}$ EDTA) and incubated at $68^{\circ} \mathrm{C}$ for $1 \mathrm{~h}$. The annealed DNA fragments were ligated into the $\mathrm{pSilencer}{ }^{\mathrm{TM}}$ 4.1-CMV hygromycin vector. After amplification, the vectors were DNA sequenced, termed H1-shRNA and H2-shRNA, and used in our subsequent experiments.

Next, $2 \times 10^{5} \mathrm{HCC}$ cells were plated in a $35-\mathrm{mm}$ dish, grown for $24 \mathrm{~h}$, and transfected with the H1-shRNA and H2-shRNA or negative control vector (Ambion). The transfected cells were selected under $0.8 \mathrm{mg} / \mathrm{ml}$ hygromycin B (BD Biosciences, San Jose, CA, USA) for 2 days and cloned by dilution and seeding into 96-well plates. Expanded cell clones were then characterized.
Cell viability assay. The colorimetric 3-(4,5-dimethylthiazol2-yl)-2,5-diphenyltetrazolium bromide (MTT) assay was used to assess changes in cell viability as previously described with some modifications (13). Briefly, the negative control cells and H1-shRNA and H2-shRNA transfected cells were grown to $70-80 \%$ confluency, trypsinized, seeded at 5,000 cells/well into a 96-well plates, and then cultured for 7 days. MTT was added to the cells for the cell viability assay each day for 7 days. Furthermore, these cells also were treated with increasing concentrations of cisplatin (DDP) for an additional $24 \mathrm{~h}$. After that, MTT was added to each well, and the cells were further incubated at $37^{\circ} \mathrm{C}$ for $3 \mathrm{~h}$ to allow formation of the purple formazan precipitate, which was then solubilized in dimethylsulfoxide. The optical density (OD) at $570 \mathrm{~nm}$ was determined in a microtiter plate reader (Tecan, San Jose, CA, USA). Cell viability relative to control cells (100\%) was determined. Experiments were repeated at least four times. The $\mathrm{IC}_{50}$ values for the different groups were analyzed using SPSS software version 13.0 (SPSS Inc., Chicago, IL, USA).

Flow cytometric detection of cell cycle distribution. Both the negative control and gene-transfected cells $\left(1-2 \times 10^{6}\right)$ were harvested, washed twice using phosphate-buffered saline (PBS) and resuspended in $200 \mu \mathrm{l}$ PBS. The cells were fixed in $4 \mathrm{ml}$ cold $75 \%$ ethanol at $4^{\circ} \mathrm{C}$ for a minimum of $4 \mathrm{~h}$, then washed twice in PBS and resuspended in $500 \mu 1$ PBS. After that, the cells were stained by addition of $200 \mu$ l propidium iodide $(50 \mu \mathrm{g} / \mathrm{ml})$ along with $20 \mu \mathrm{l}$ RNase $(1 \mathrm{mg} / \mathrm{ml})$ (both from Sigma-Aldrich, St. Louis, MO, USA) in a $37^{\circ} \mathrm{C}$ water bath for $20 \mathrm{~min}$. Cell cycle distribution was determined by fluorescence-activated cell sorting (FACS) and analyzed using CellQuest software (BD Biosciences).

Flow cytometric detection of apoptosis. Both the negative control and H1-siRNA and H2-siRNA transfected cells were grown to $70-80 \%$ confluency and then exposed to $5 \mu \mathrm{g} / \mathrm{ml}$ Hoechst 33342,250 nM MitoTracker Red, and $1 \mu \mathrm{M}$ YO-PRO-1 in Hanks balanced salt solution supplemented with $10 \%$ FCS for $20 \mathrm{~min}$ at room temperature. The membrane of apoptotic cells but not live cells was permeated by the YO-PRO-1. Hoechst 33342 is a specific stain for double-stranded DNA, whereas MitoTracker Red is concentrated by active mitochondria in living cells. Fluorescence of stained cells was then detected using a fluorescence microscope (Olympus, Japan). The proportion of apoptotic cells (apoptotic ratio, AR) was calculated using the formula: $A R=A / T$, where $A$ is the number of apoptotic cells [YO-PRO-1-positive (green) and Hoechst 33342-positive (blue) but MitoTracker Red-negative (red)] and $\mathrm{T}$ is the total cell (Hoechst-positive) count. Up to 20 images of each sample were acquired and analyzed in two different experiments as described in a previous study (14).

Protein extraction and western blotting. Cells were lysed in buffer A (10 mM Tris-HCl, $150 \mathrm{mM} \mathrm{NaCl,} 1 \%$ Triton X-100, $5 \mathrm{mM}$ EDTA, $10 \mathrm{mM}$ sodium pyrophosphate, $10 \%$ glycerol, $0.1 \% \mathrm{Na}_{3} \mathrm{VO}_{4}$ and $50 \mathrm{mM} \mathrm{NaF}, \mathrm{pH}$ 7.4) followed by centrifugation at $10,000 \mathrm{x} \mathrm{g}$ for $10 \mathrm{~min}$ at $4^{\circ} \mathrm{C}$ to remove cell debris. Lysates were then assayed for total protein using the bicinchoninic acid (BCA) assay (Pierce, Rockford, IL, USA). Lysates were boiled at $95^{\circ} \mathrm{C}$ for $15 \mathrm{~min}$ in solution 


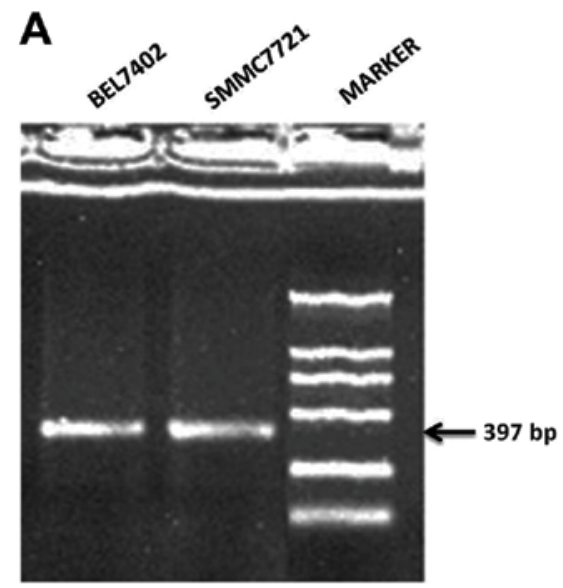

B
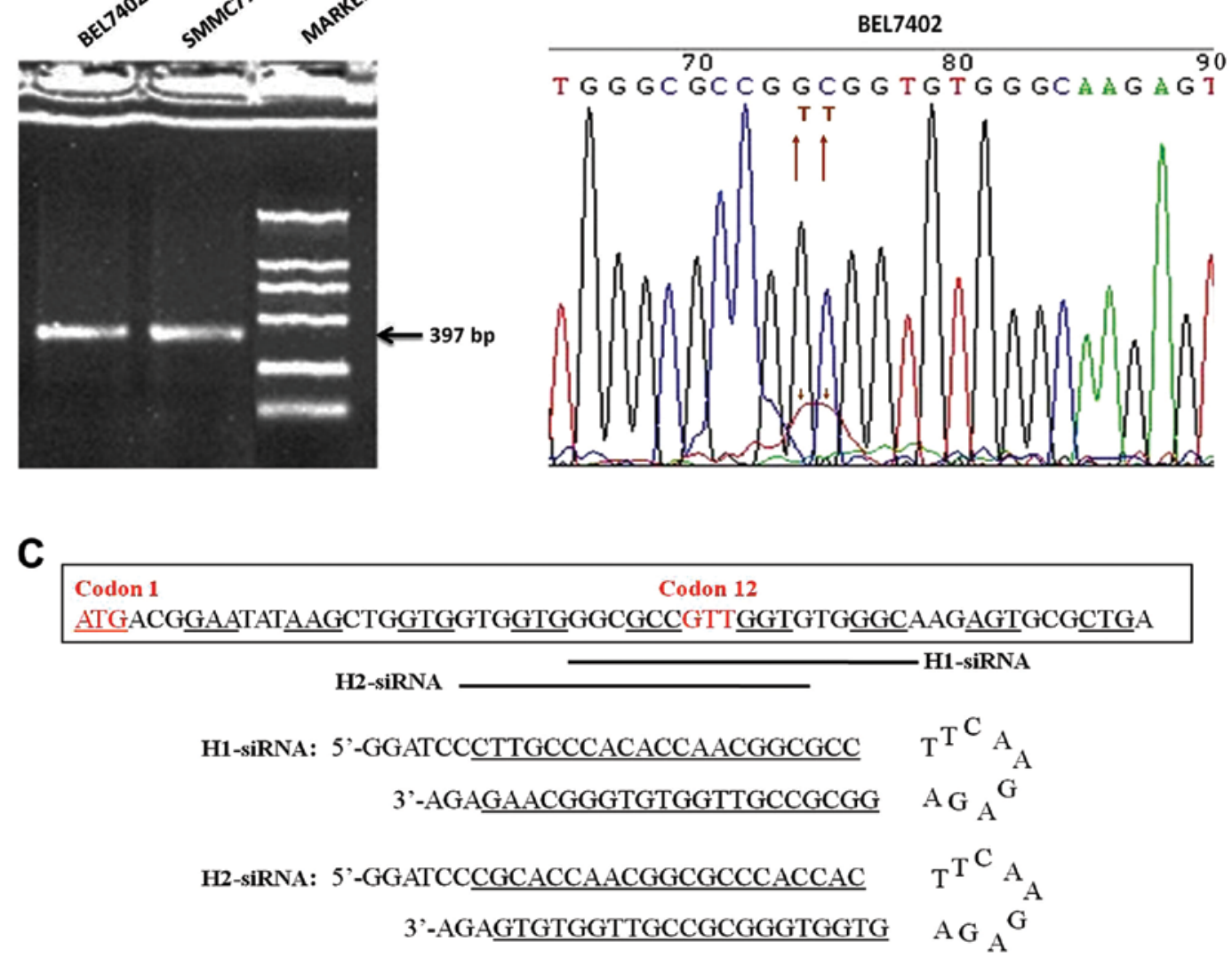

Figure 1. Detection of the H-Ras mutation in the HCC cell lines and design of the shRNA constructs. (A) RT-PCR analysis of H-Ras cDNA in the BEL7402 and SMMC7721 cells. (B) DNA sequence of the H-Ras mutation in the BEL7402 cells. (C) H-Ras cDNA sequence and the two shRNAs that target oncogenic mutation codon 12 are illustrated schematically. H1-shRNA and H2-shRNA were designed to target the 216-236 and 210-230 sequences of H-Ras mRNA (NM_001130442.1 CDS: 189-758), respectively.

containing $100 \mathrm{mM}$ DTT, then separated by sodium dodecyl sulfate-polyacrylamide gel electrophoresis (SDS-PAGE), and transferred onto polyvinylidene fluoride (PVDF) membranes (Millipore, Darmstadt, Germany). For western blotting, the membranes were blocked for $1 \mathrm{~h}$ in PBS-Tween-20/5\% skim milk or bovine serum albumin (BSA) and then incubated overnight at $4^{\circ} \mathrm{C}$ with the corresponding primary antibodies. After three washes in PBS-Tween-20, the blots were incubated with a horseradish peroxidase-conjugated secondary antibody for $1 \mathrm{~h}$ and washed three times with PBS-Tween-20. Protein bands were visualized using an enhanced chemiluminescence (ECL) reagent (Pierce) and exposed to an X-OMAT film (Kodak, Rochester, NY, USA).

Murine tumor allograft assay. All experiments conformed to the Animal Care and Use Guidelines of the Institute for Nutritional Sciences (Shanghai, China). Briefly, 4- to 6-week old female BALB/c athymic nude mice were obtained from Shanghai Experimental Animal Center (Shanghai, China) and subcutaneously injected with a suspension containing $5 \times 10^{6}$ negative control and H1-siRNA and H2-siRNA transfected cells. One week after cell implantation, DDP (4 mg/ $\mathrm{kg}$ ) was injected intraperitoneally into the tumor-bearing mice every 4 days for 3 weeks (between days 8 and 31). General clinical observations of the mice, including determination of body weight and tumor growth, were conducted twice a week. We measured two perpendicular diameters of the xenograft using calipers to determine tumor size. Tumor mass was estimated using the formula: $\left(a \mathrm{xb}^{2}\right) / 2$, where ' $\mathrm{a}$ ' is the longest diameter and ' $b$ ' is the shortest diameter as described in previous studies $(15,16)$. All mice were euthanized by $\mathrm{CO}_{2}$ asphyxiation 2 weeks after the last injection, and the tumor xenografts were removed and processed for evaluation.

Statistical analysis and data reproducibility. All of the experiments were repeated at least three times. Results are presented as means \pm standard deviations (SDs). Statistical evaluation was performed, where appropriate, using the Student's t-test or analysis of variance (ANOVA) followed by correction with Bonferroni's t-test. A p-value $\leq 0.05$ was considered to indicate a statistically significant result, and all analyses were carried out using SPSS software version 13.0.

\section{Results}

Knockdown of mutant $H$-Ras expression using shRNA in HCC cell lines. In the present study, we first assessed the H-Ras V12 mutation in a HCC cell line and found that BEL7402 cells had a mutation of V12 (Val/Gly) and wild-type H-Ras, whereas SMMC7721 cells expressed only wild-type H-Ras (Fig. 1). Thus, we utilized BEL7402 cells in further experiments to assess the effect of the H-Ras V12 mutant knockdown on the 


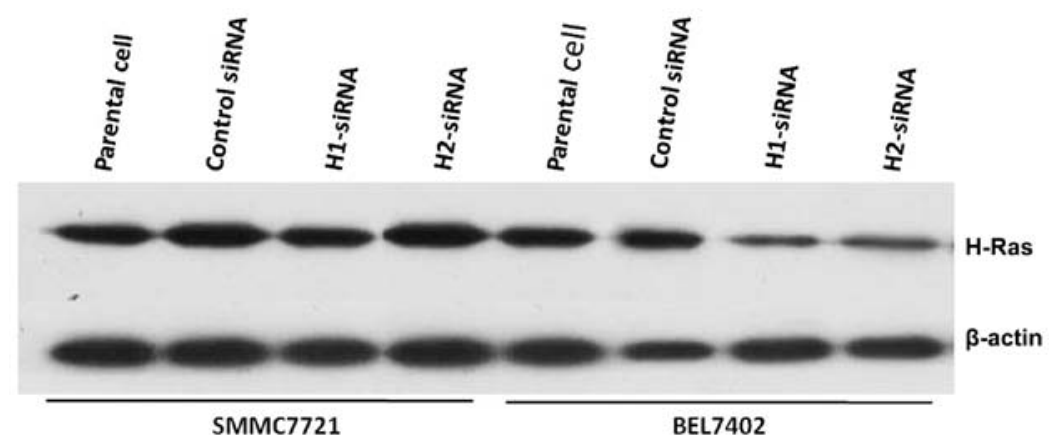

Figure 2. Knockdown of mutated H-Ras protein expression in BEL7402 and SMMC7721 cells using H1-shRNA and H2-shRNA compared to the negative control vector.

A

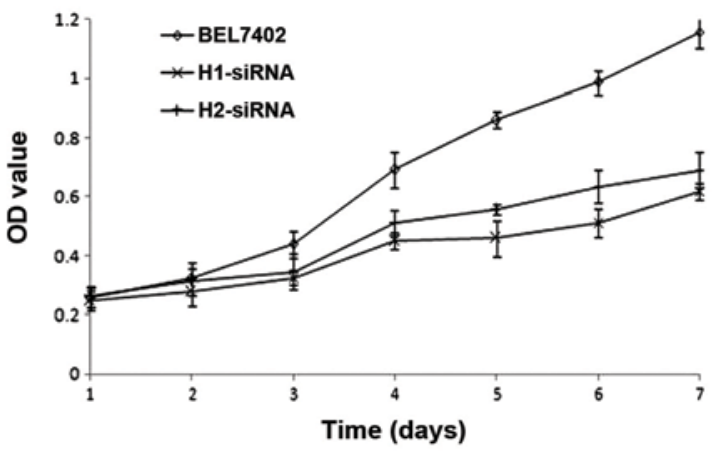

C

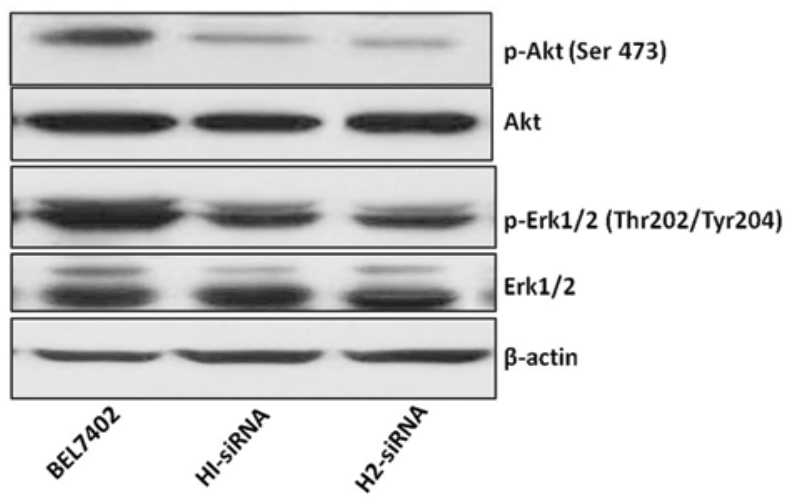

B

$\mathrm{GO} / \mathrm{G} 1=32.7 \%$

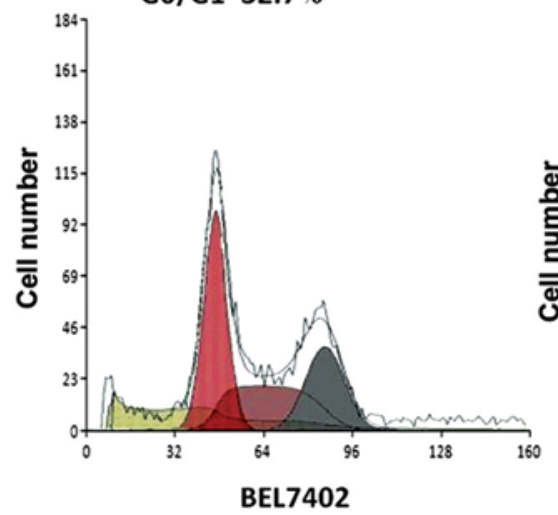

$\mathrm{G} 0 / \mathrm{G} 1=46.2 \%$

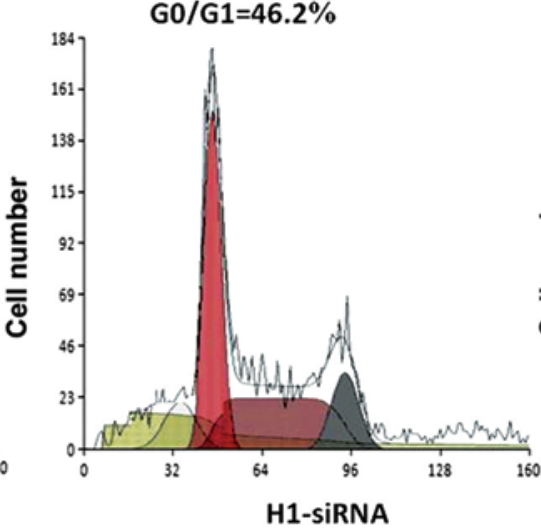

G0/G1 $=47.8 \%$

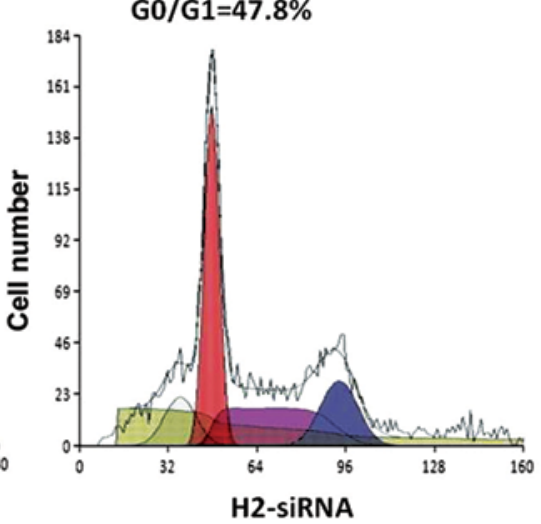

Figure 3. Cell viability and cell cycle analyses of BEL7402 cells expressing H1-shRNA and H2-shRNA. (A) MTT assay. (B) Flow cytometry. Cell cycle distribution was analyzed using flow cytometry with propidium iodide staining. (C) Western blot analysis of Akt, p-Akt (Ser473), Erk1/2 and p-Erk1/2 (Thr202/ Tyr204) expression.

regulation of $\mathrm{HCC}$ cell viability and apoptosis in vitro as well as HCC cell xenograft tumor formation and growth in nude mice.

We then stably knocked down H-Ras expression in BEL7402 cells, and as shown in Fig. 2, the two shRNA constructs specifically silenced H-Ras expression in the BEL7402 cells, but did not affect wild-type Ras expression in the SMMC7721 cells.

Knockdown of mutant $H$-Ras expression reduces $H C C$ cell viability and arrests cells at the G0/Gl phase of the cell cycle. Next, we assessed the change in cell viability in
BEL7402 cells after knockdown of mutant $H$-Ras expression. Introduction of H1-shRNA and H2-shRNA into BEL7402 cells led to a significant reduction in cell viability compared to that in the parental and negative control cells (Fig. 3A). Starting on the 3rd day, the reduction became much more obvious $(p<0.01)$. These results suggest that knockdown of $\mathrm{H}$-Ras mutant protein expression had significant specificity and that the H-Ras V12 mutant played an important role in BEL7402 cell growth.

Furthermore, we also assessed the change in cell cycle distribution in these cells and found that the percentages of BEL7402 cells in the G0/G1 phase of the cell cycle after 


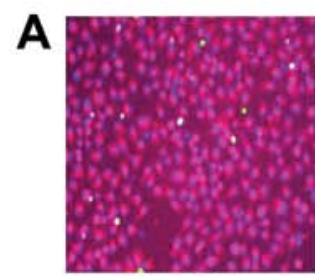

BEL7402

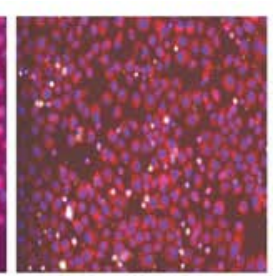

HI-siRNA

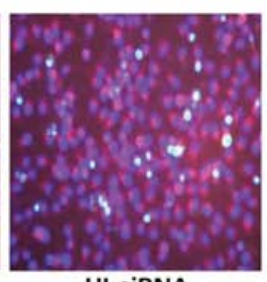

HI-siRNA

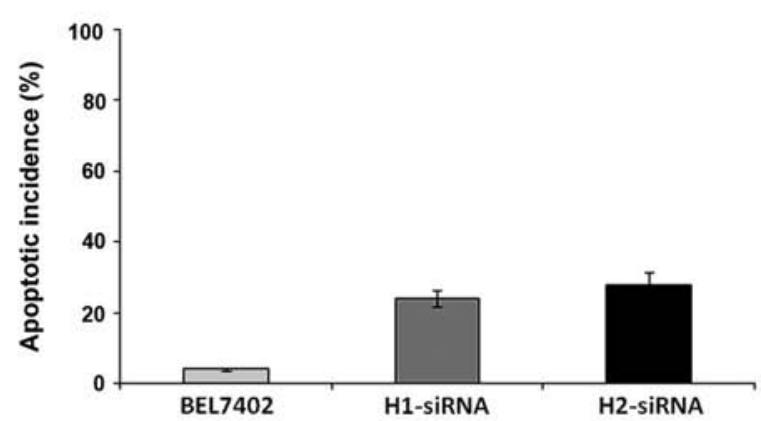

B
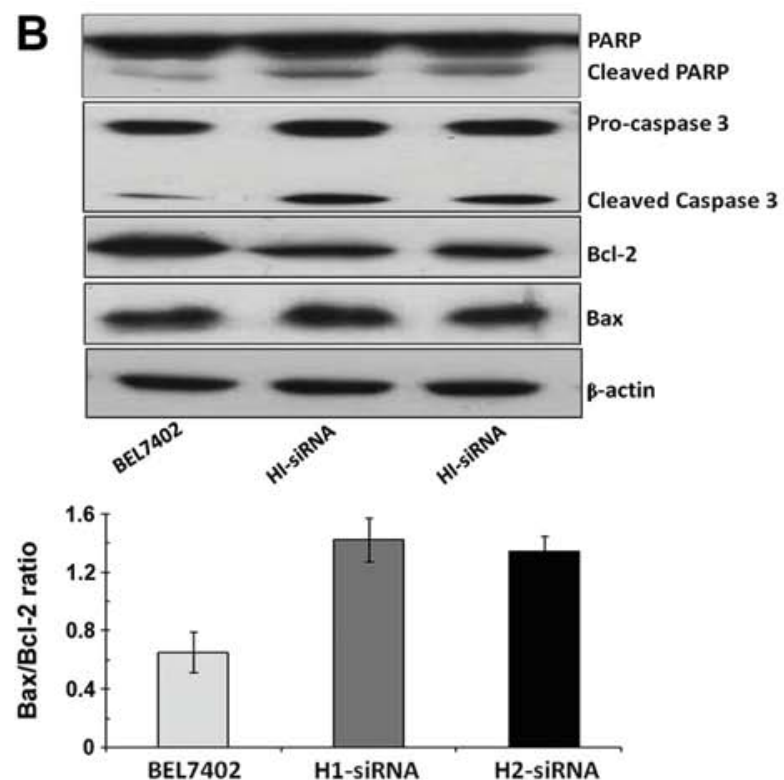

Figure 4. Effect of H1-shRNA and H2-shRNA on the regulation of cell apoptosis in BEL7402 cells. (A) BEL7402 cells stably expressing H1-shRNA and H2-shRNA were stained by Hoechst 33342, MitoTracker Red and YO-PRO-1 for detection of apoptosis. The proportion of apoptotic cells (AR, apoptotic ratio) was calculated using the formula $\mathrm{AR}=\mathrm{A} / \mathrm{T}$ where $\mathrm{A}$ is the number of apoptotic cells [YO-PRO-1-positive (green) and Hoechst 33342-positive (blue) but MitoTracker Red-negative (red)] and $\mathrm{T}$ is the total cell (Hoechst-positive) count. (B) Western blot analysis of the ratio of Bax/Bcl-2. Column, mean ( $\mathrm{n}=3$ ); bar, standard error (SE).

transfection with H1-shRNA and H2-shRNA (46.2 and 47.8\%, respectively) were higher compared to $32.7 \%$ in the negative control cells $(\mathrm{p}<0.01$; Fig. 3B). This induction was associated with a decrease in the percentages of cells in the $S$ and G2/M phases of the cell cycle.

In addition, we also analyzed the changes in expression of H-RAs-related genes (Fig. 3C). For example, expression of phospho-Akt was significantly decreased in the BEL7402 cells after they were stably transfected with H1-shRNA and H2-shRNA compared to the negative control cells. However, there was only a mild decrease in phospho-Erk expression in these cells. These data demonstrated that H-Ras V12 knockdown retarded cell proliferation by keeping the cells in the G0/G1 phase through decreasing phosphorylation of Akt and Erk proteins.

Knockdown of mutant H-Ras expression induces BEL7402 cell apoptosis. Next, we assessed the effects of H1-shRNA and H2-shRNA-induced H-Ras V12 knockdown on the regulation of HCC cell apoptosis and expression of apoptotic pathway genes. The data showed that more BEL7402 cells transfected with H1-shRNA or H2-shRNA underwent apoptosis compared to the negative control cells (Fig. 4A). The proportion of apoptotic BEL7402 cells was 24 and $28 \%$ in the H1-siRNA and H2-siRNA transfected cells, respectively, compared to $4 \%$ in the control cells.

Western blot data showed that H-Ras V12 silencing in BEL7402 cells led to the activation of several apoptotic proteins (e.g., caspase-3 and its substrate, PARP; Fig. 4B) and a dramatic reduction in Bcl-2 levels. Although expression of the pro-apoptotic protein Bax showed no obvious change, the ratio of $\mathrm{Bax} / \mathrm{Bcl}-2$ was elevated. Collectively, these data suggest that H-Ras V12 knockdown in BEL7402 cells could induce cancer cell death through downregulation of Bcl-2 expression.

H-Ras V12 knockdown increases the chemosensitivity of $H C C$ cells in vitro and in vivo. Furthermore, we explored the increasing chemosensitivity of HCC cells after H-Ras V12 silencing in vitro and in vivo using nude mouse xenografts. As shown in Fig. 5A, DDP treatment caused a significant retardation in BEL7402 cell growth after transfection with H1-shRNA or H2-shRNA and resulted in $\mathrm{IC}_{50}$ values of 0.51 and $0.68 \mu \mathrm{M}$, respectively. The sensitivity toward DDP increased by 7.19- and 5.39-fold in the H1-shRNA and H2-shRNA transfected cells, respectively, compared to the negative control cells. The results of a flow cytometric apoptotic assay showed significantly increased tumor apoptosis after DDP treatment in the H-Ras V12-silenced cells compared to the negative control cells. These data suggest that suppression of H-Ras mutant oncoprotein could enhance the sensitivity of HCC cells to DDP therapy. Western blot data showed that a 24-h DDP treatment led to increased cleavage of caspase-3 and PARP in BEL7402 cells in the H-Ras V12-silenced HCC cells in a concentration-dependent manner (Fig. 5C). However, at any given concentration, DDP exposure resulted in significantly increased levels of proteolytic cleavage of caspase-3 and PARP in cells with H-Ras V12 knockdown compared to the negative control BEL7402 cells. These results indicate that the H-Ras V12-silenced cells were more susceptible to DDP treatment. At the gene level, Bcl-2 and Bax were not significantly altered in any group with increased DDP concentration, whereas expression of Puma protein was increased significantly in a dose-dependent manner. In addition, we assessed the effects of increasing chemosensitivity of HCC cells after H-Ras V12 silencing in in vivo nude mouse xenografts. As shown 
A

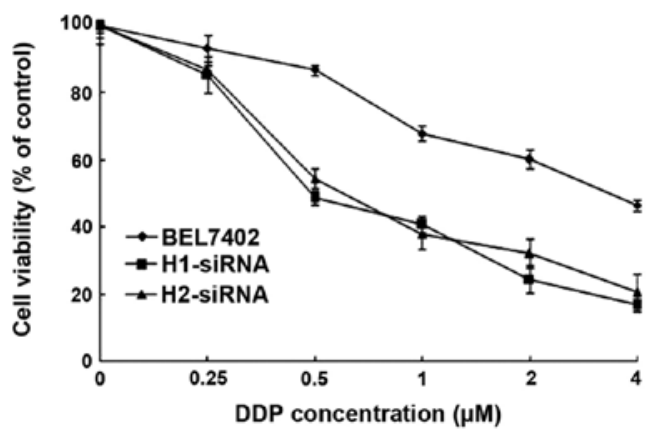

B

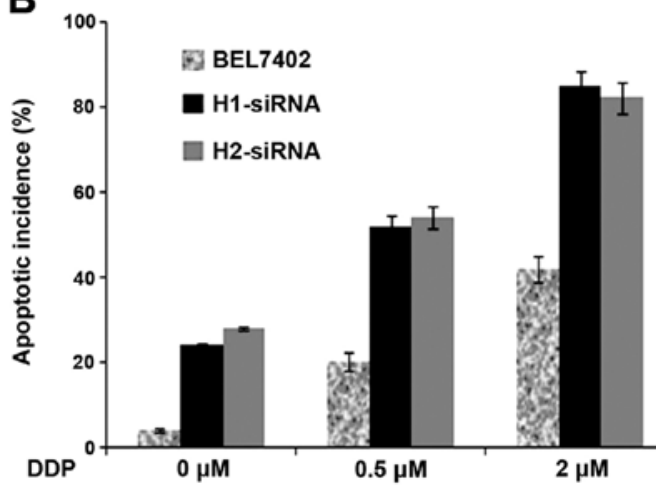

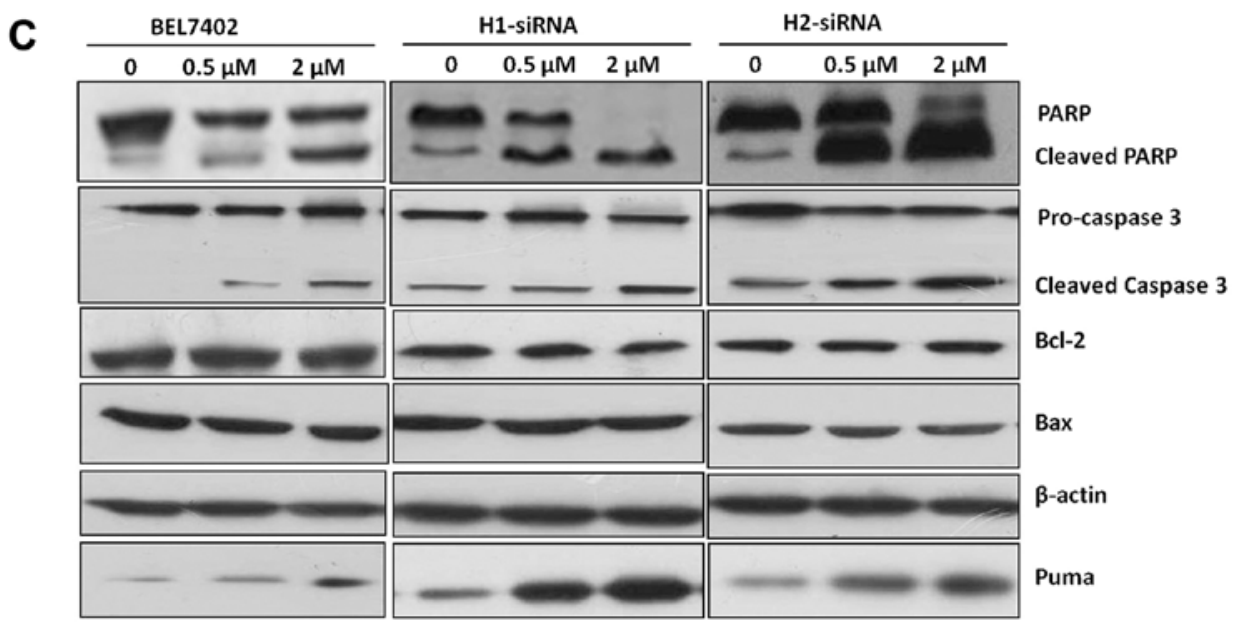

Figure 5. Increase in the chemosensitivity of HCC cells to cisplatin treatment after H-Ras V12 silencing. (A) Cell viability was reduced after DDP treatment in BEL7402 cells and cells expressing H1-siRNA or H2-siRNA. (B) The proportion of apoptotic cells (apoptotic incidence) was detected using YO-PRO-1 staining. (C) Western blot analysis of apoptotic pathway-related genes (Bax, Bcl-2, PARP, caspase-3 and Puma) after cells were exposed to DDP treatment. $\beta$-actin was used as a loading control.

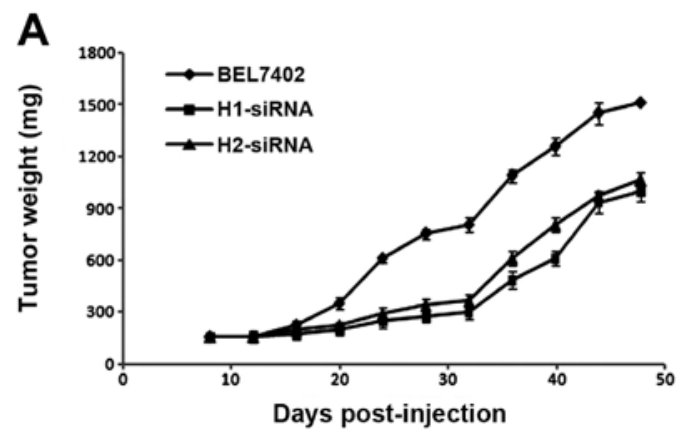

$c$

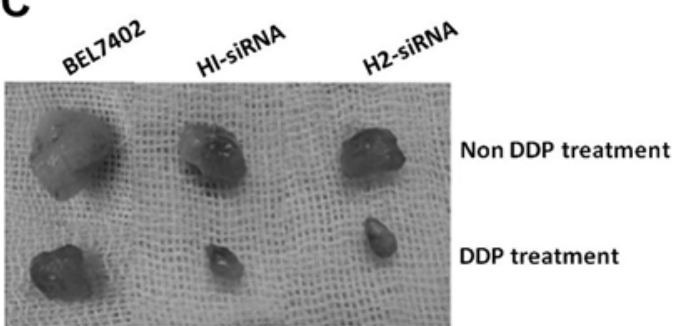

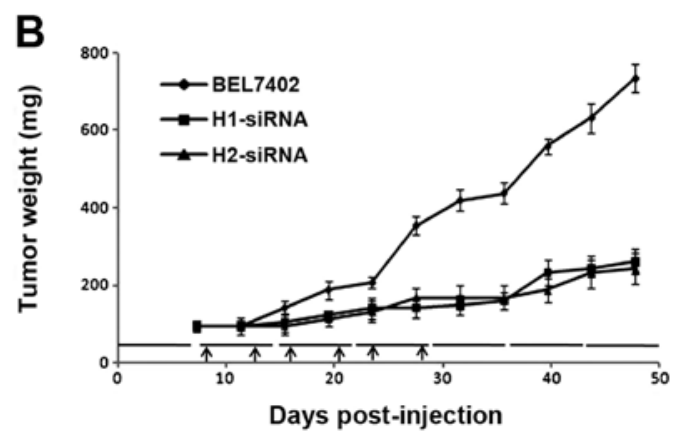

Figure 6. Effect of H-Ras V12 knockdown on the regulation of HCC cell xenograft formation and growth. Nude mice were implanted with $5 \times 10^{6}$ cells on day 0 in each group. (A) Tumor growth of BEL7402 cells after stable transfection with H1-shRNA or H2-shRNA was observed after injection. (B) Beginning 8 days after tumor cell implantation, tumor-bearing mice were treated with DDP ( $4 \mathrm{mg} / \mathrm{kg}$; i.p.) for 3 weeks at 4 consecutive days/week (thin arrow). (C) Tumor sizes were measured using calipers. Columns, mean; bars, SD.

treatment by the end of the experiments. However, intraperitoneal administration of DDP at $4 \mathrm{mg} / \mathrm{kg}$ led to substantial reductions in tumor growth by 81 and $77 \%$ in the H1-shRNA in Fig. 6A, tumor xenografts transplanted from the H-Ras V12-silenced cells exhibited mild growth inhibition compared to the tumors from the negative control cells without DDP 
and H2-shRNA transfected tumor xenografts, respectively, compared to a $37 \%$ reduction in mice bearing negative control tumor cells ( $\mathrm{p}<0.01$; Fig. 6B and $\mathrm{C}$ ).

\section{Discussion}

The human Ras family of proteins consists of three members ( $\mathrm{H}-, \mathrm{N}-$ and $\mathrm{K}-\mathrm{Ras})$, and mutation of these can transform normal cells into neoplasms $(17,18)$. The most commonly reported activating Ras mutations occur at codons 12,13 and 61. In human HCC, Ras point mutations have been frequently observed in N-Ras, with an incidence of $\sim 30 \%$, whereas H-Ras mutations are found in $29.3-71.01 \%$ of HCC cases $(6,19,20)$. In the present study, we first assessed the mutation of H-Ras in HCC cell lines and determined the effects of H-Ras V12 knockdown on the regulation of HCC cell viability, apoptosis and gene expression in vitro and in nude mouse xenografts. We found that the H-Ras mutation occurred at codon 12 in BEL7402 cells with a change from GGC(Gly) to GTT(Val). BEL7402 cells also expressed wild-type H-Ras, which provided an ideal model system to investigate the effects of knockdown of mutated H-Ras V12 expression with maintenance of normal functions of wild-type H-Ras in the cells. Indeed, our current data showed that knockdown of mutated H-Ras expression reduced BEL7402 cell viability and induced apoptosis by arresting the cells at the G0/G1 phase of cell cycle. At the gene level, expression of phospho-Akt was also significantly decreased, whereas phospho-Erk expression was mildly decreased in stably H1-shRNA and H2-shRNA transfected BEL7402 cells. Moreover, several apoptotic proteins (e.g., caspase-3 and its substrate, PARP) were also activated, and the ratio of $\mathrm{Bax} / \mathrm{Bcl}-2$ was increased. In addition, the sensitivity of H1-shRNA and H2-shRNA transfected BEL7402 cells to DDT treatment was increased by 7.19- and 5.39-fold, respectively, compared to the negative control cells. Intraperitoneal administration of cisplatin resulted in a substantial reduction in tumor xenograft growth. Thus, the data from the present study demonstrated that knockdown of mutated H-Ras V12 expression induced chemosensitivity of HCC cells to cisplatin treatment in vitro and in nude mouse xenografts. Future studies will evaluate the clinical effects of H1-shRNA and H2-shRNA transfection.

One major problem in the design of a sequence-specific anti-Ras therapy is that wild-type and mutant Ras differ only by a single codon. The shRNA interference technique is characterized by extraordinary sequence specificity and may provide a useful tool for cancer therapy against mutant Ras. Indeed, the use of shRNA in cancer treatment has been reported extensively. For example, Yang et al showed that tumor transformation and growth were decreased after knockdown of H-Ras V12 expression using a retrovirus-mediated shRNA in human ovarian cancer (12). Fleming et al used a retroviral RNAi to inhibit expression of mutated K-Ras V12, while leaving other Ras isoforms unaffected (21). The present study showed that H1-shRNA and H2-shRNA were able to specifically target mutated H-Ras V12 expression, but not affect wild-type H-Ras expression in SMMC7721 cells.

In the present study, we also showed changes in cell cycle distribution, apoptosis and gene expression in HCC cells after knockdown of H-Ras V12 expression. The Ras function is dependent on the cycle (switch) between active (GTP-bound) and inactive (GDP-bound) forms of Ras protein, whereas active mutant Ras protein is usually locked in the active state and thus, delivers uncontrolled cell proliferative signals. Activated Ras protein stimulates downstream effectors, such as the Raf-MEK-MAPK and PI3K-Akt pathways (22). For example, activation of the p44/42 MAPK (Erk1/2) leads to stimulation of several transcription regulators and key cell cycle regulatory proteins, such as D-type cyclins, and in turn enables the cells to progress through the G1 phase of the cell cycle $(23,24)$. Akt is involved in cell cycle regulation by preventing GSK-3 $\beta$ mediated phosphorylation and degradation of cyclin D1 (18), and by negatively regulating the cyclin-dependent kinase inhibitors $\mathrm{p} 27^{\mathrm{Kip}}(20)$ and $\mathrm{p} 21^{\mathrm{Waf} 1 / \mathrm{CIP} 1}(25,26)$. Thus, in the present study, we observed that phosphorylation of Akt and Erk was decreased dramatically in BEL7402 cells after transfection with H-Ras V12 shRNA, which contributed to cell cycle arrest at the G0/G1 phase. Furthermore, we also found that H-Ras V12 knockdown in BEL7402 cells caused cleavage of caspase- 3 and PARP, which was accompanied by a reduction in Bcl-2 levels and elevation of the ratio of Bax/Bcl-2 for induction of HCC cell apoptosis. Indeed, Pugazhenthi et al showed that PI3K and Akt phosphorylation led to an increase in CREB-driven Bcl-2 promoter activity and thus downregulated Bcl-2 expression (27). Therefore, reduction in Bcl-2 expression could be attributed to its transcriptional inhibition controlled by the downregulation of the Akt-CREB pathway in cells with H-Ras V12 knockdown.

The diverse consequences of specific knockdown of mutant Ras, such as tumor growth, angiogenesis, metabolism and metastasis, have been demonstrated in several studies $(12,21,28)$. However, induction of chemosensitivity and the corresponding molecular mechanisms after knockdown of mutant Ras have not been explored. In the present study, we assessed changes in chemosensitivity of BEL7402 cells after knockdown of mutated H-Ras V12 expression to cisplatin treatment (a widely used chemotherapeutic drug for cancer patients). We found that compared to the negative control vector-transfected $\mathrm{HCC}$ cells, cisplatin treatment reduced cell viability and the $\mathrm{IC}_{50}$ values in the $\mathrm{H}$-Ras V12-knockdown HCC cells, yet induced apoptosis in HCC cells in vitro and tumor growth in the athymic xenograft mouse model. These data revealed that the increased sensitivity to cytotoxic drugs is attributed to disruption of the balance between anti-apoptotic Bcl-2 and pro-apoptotic Bax after knockdown of mutant H-Ras V12 expression. This finding is novel, and to date, no similar studies have been reported. At the gene level, we found that activation of caspase-3 and PARP enzymes occurred in a cisplatin dose-dependent manner, although there was no alteration of Bcl-2 and Bax expression. These data suggest that the cytotoxicity of cisplatin in vivo may have other modulating mechanisms. Indeed, the present data showed that cisplatin treatment significantly increased the level of p53 upregulated modulator of apoptosis (Puma) protein in a dose-dependent manner. Puma was identified as a novel BH3-only protein (29) and plays a critical role in the regulation of p53 activity in the p53 tumor suppressor pathway $(30,31)$. Functionally, expression of Puma- $\alpha$ can be induced by p53 protein, and then Puma- $\alpha$ binds to Bcl-2 and Bcl-xL, both of which are localized in the mitochondria, and in turn promotes cyctochrome $c$ release 
and apoptosis (32). Jiang et al demonstrated that Puma- $\alpha$ is upregulated and bound to $\mathrm{Bcl}-\mathrm{xL}$ in $\mathrm{p} 53$-mediated renal cell apoptosis during cisplatin-induced nephrotoxicity (30). Tsuruya et al also showed that Puma- $\alpha$ plays an essential role in p53 activation-mediated, cisplatin-induced renal tubular cell death (33). These studies suggest that Puma- $\alpha$ could be transcribed by $\mathrm{p} 53$ during cisplatin treatment.

In summary, our present data demonstrated that our specifically designed H-Ras V12 shRNAs were able to knock down expression of mutated H-Ras V12, but not expression of wild-type H-Ras, and that knockdown of mutated H-Ras V12 expression promoted chemosensitivity of HCC cells to cisplatin treatment in vitro and in vivo. Mechanistically, the effect involved a reduction in the Bcl-2 level, whereas cisplatin treatment increased Puma- $\alpha$ expression. Further studies will assess the potential translation of our current findings into clinical trials.

\section{Acknowledgements}

This study was supported in part by grants from the China Medical Board (no. 06-837), and the Hebei Science and Technology Research and Development Guidance Program (no. 072761710). This manuscript was edited and proofread by Medjaden Bioscience Limited.

\section{References}

1. Jemal A, Bray F, Center MM, Ferlay J, Ward E and Forman D Global Cancer Statistics. CA Cancer J Clin 61: 69-90, 2011.

2. Saxena N, Lahiri SS, Hambarde S and Tripathi RP: RAS: target for cancer therapy. Cancer Invest 26: 948-955, 2008.

3. Boguski MS and McCormick F: Proteins regulating Ras and its relatives. Nature 366: 643-654, 1993.

4. Bos JL: ras oncogenes in human cancer: a review. Cancer Res 49 : 4682-4689, 1989.

5. Bollag G and McCormick F: Regulators and effectors of ras proteins. Annu Rev Cell Biol 7: 601-632, 1991.

6. Sui G, Ma X, Liu S, Niu H and Dong Q: Study of the correlation between $\mathrm{H}$-ras mutation and primary hepatocellular carcinoma. Oncol Lett 4: 779-782, 2012.

7. Lim KH and Counter CM: Reduction in the requirement of oncogenic Ras signaling to activation of PI3K/AKT pathway during tumor maintenance. Cancer Cell 8: 381-392, 2005.

8. Rotblat B, Ehrlich M, Haklai R and Kloog Y: The Ras inhibitor farnesylthiosalicylic acid (Salirasib) disrupts the spatiotemporal localization of active Ras: a potential treatment for cancer. Methods Enzymol 439: 467-489, 2008.

9. Reuter C, Morgan M and Bergmann L: Targeting the Ras signaling pathway: a rational, mechanism-based treatment for hematologic malignancies? Blood 96: 1655-1669, 2000.

10. Méndez-Sánchez N, Vásquez-Fernández F, Zamora-Valdés D and Uribe M: Sorafenib, a systemic therapy for hepatocellular carcinoma. Ann Hepatol 7: 46-51, 2008.

11. Njoroge FG, Doll RJ, Vibulbhan B, et al: Discovery of novel nonpeptide tricyclic inhibitors of Ras farnesyl protein transferase. Bioorg Med Chem 5: 101-113, 1997.

12. Yang G, Thompson JA, Fang B and Liu J: Silencing of H-ras gene expression by retrovirus-mediated siRNA decreases transformation efficiency and tumor growth in a model of human ovarian cancer. Oncogene 22: 5694-5701, 2003.
13. Hansen MB, Nielsen SE and Berg K: Re-examination and further development of a precise and rapid dye method for measuring cell growth/cell kill. J Immunol Methods 119: 203-210, 1989.

14. Paoluzzi L, Gonen M, Bhagat G, et al: The BH3-only mimetic ABT-737 synergizes the antineoplastic activity of proteasome inhibitors in lymphoid malignancies. Blood 112: 2906-2916, 2008.

15. Wang H, Rayburn ER, Wang W, Kandimalla ER, Agrawal S and Zhang R: Chemotherapy and chemosensitization of non-small cell lung cancer with a novel immunomodulatory oligonucleotide targeting Toll-like receptor 9. Mol Cancer Ther 5: 1585-1592, 2006.

16. Sepp-Lorenzino L, Rands E, Mao X, et al: A novel orally bioavailable inhibitor of kinase insert domain-containing receptor induces antiangiogenic effects and prevents tumor growth in vivo. Cancer Res 64: 751-756, 2004.

17. Crowder C, Kopantzev E, Williams K, Lengel C, Miki T and Rudikoff S: An unusual H-Ras mutant isolated from a human multiple myeloma line leads to transformation and factor-independent cell growth. Oncogene 22: 649-659, 2003.

18. Johnston SR: Farnesyl transferase inhibitors: a novel targeted tnerapy for cancer. Lancet Oncol 2: 18-26, 2001.

19. Tang Z, Qin L, Wang X, et al: Alterations of oncogenes, tumor suppressor genes and growth factors in hepatocellular carcinoma: with relation to tumor size and invasiveness. Chin Med J 111: 313-318, 1998.

20. Iida M, Iwata $\mathrm{H}$, Inoue $\mathrm{H}$, Enomoto $\mathrm{M}$, Horie $\mathrm{N}$ and Takeishi $\mathrm{K}$ : Correlation between Bcl-2 overexpression and $H$-ras mutation in naturally occurring hepatocellular proliferative lesions of the B6C3F1 mouse. Toxicol Sci 56: 297-302, 2000.

21. Fleming JB, Shen GL, Holloway SE, Davis M and Brekken RA: Molecular consequences of silencing mutant K-ras in pancreatic cancer cells: justification for K-ras-directed therapy. Mol Cancer Res 3: 413-423, 2005.

22. Adjei AA: Blocking oncogenic Ras signaling for cancer therapy. J Natl Cancer Inst 93: 1062-1074, 2001.

23. Roberts PJ and Der CJ: Targeting the Raf-MEK-ERK mitogenactivated protein kinase cascade for the treatment of cancer. Oncogene 26: 3291-3310, 2007.

24. Friday BB and Adjei AA: Advances in targeting the Ras/Raf/ MEK/Erk mitogen-activated protein kinase cascade with MEK inhibitors for cancer therapy. Clin Cancer Res 14: 342-346, 2008.

25. Diehl JA, Cheng M, Roussel MF and Sherr CJ: Glycogen synthase kinase- $3 \beta$ regulates cyclin D1 proteolysis and subcellular localization. Genes Dev 12: 3499-3511, 1998.

26. Gesbert F, Sellers WR, Signoretti S, Loda M and Griffin JD: BCR/ABL regulates expression of the cyclin-dependent kinase inhibitor $\mathrm{p} 27^{\mathrm{Kipl}}$ through the phosphatidylinositol 3-kinase/AKT pathway. J Biol Chem 275: 39223-39230, 2000.

27. Pugazhenthi S, Nesterova A, Sable C, et al: Akt/protein kinase B up-regulates Bcl-2 expression through cAMP-response elementbinding protein. J Biol Chem 275: 10761-10766, 2000.

28. Bernhard EJ: Pancreatic cancer inhibition by specific knockdown of K-ras mutant allele expression. Cancer Biol Ther 6: 293-294, 2007.

29. Ming L, Wang P, Bank A, Yu J and Zhang L: PUMA dissociates $\mathrm{Bax}$ and $\mathrm{Bcl}-\mathrm{X}_{\mathrm{L}}$ to induce apoptosis in colon cancer cells. J Biol Chem 281: 16034-16042, 2006.

30. Jiang M, Wei Q, Wang J, Du Q, Yu J, Zhang L and Dong Z: Regulation of PUMA- $\alpha$ by 553 in cisplatin-induced renal cell apoptosis. Oncogene 25: 4056-4066, 2006.

31. Hemann MT, Zilfou JT, Zhao Z, Burgess DJ, Hannon GJ and Lowe SW: Suppression of tumorigenesis by the p53 target PUMA. Proc Natl Acad Sci USA 101: 9333-9338, 2004.

32. Villunger A, Michalak EM, Coultas L, et al: p53- and druginduced apoptotic responses mediated by $\mathrm{BH} 3$-only proteins puma and noxa. Science 302: 1036-1038, 2003.

33. Tsuruya $\mathrm{K}$, Yotsueda $\mathrm{H}$, Ikeda $\mathrm{H}$, et al: Involvement of p53-transactivated Puma in cisplatin-induced renal tubular cell death. Life Sci 83: 550-556, 2008 . 